\title{
Efectos de red bull en el organismo humano
}

\author{
Red Bull Effects in Humans \\ Jocelyn Wendolyne Carmona Núñez ${ }^{1}$ *, Alma Daniela Castillo Granados ${ }^{2}$, Maricruz González \\ Osorio ${ }^{3}$, Montserrat Reyes Soto ${ }^{4}$
}

\begin{abstract}
:
The energizing drinks arise from the need to increase the energy and concentration, relieve fatigue and improve cognitive conditions before episodes of stress that is why we focus on the effects that generate these substances to the body, emphasizing the drink RED BULL. Being one of the most frequently consumed by the population and origin older. This drink containg different substances: taurine, caffeine, glucoronolactona amony others that act as a stimulant of the nervous system leading the individual to a state of alert to camy out activities.
\end{abstract}

\section{Keywords:}

Effects, energy, consumption, health.

\section{Resumen:}

Las bebidas energizantes surgen por la necesidad de aumentar la energía y la concentración, aliviar la fatiga y mejorar condiciones cognitivas ante episodios de estrés, es por eso que nos enfocamos en los efectos que generan estas sustancias al organismo haciendo énfasis en la bebida "Red Bull", siendo una de estas las más consumidas por la población y de origen más antiguo. Esta bebida contiene diferentes sustancias como cafeína, taurina, gluconorolactona entre otras, que actúan como estimuladoras del sistema nervioso llevando al individuo a un estado de alerta para poder realizar actividades.

\section{Palabras Clave:}

Efectos, energía, consumo, salud.

\section{Desarrollo}

Las bebidas energizantes son de venta libre y esta tiene una mayor prevalencia en adolescentes y adultos siendo estos sus mayores consumidores con el fin de mejorar el rendimiento intelectual, fortalecer vínculos sociales y modificar los efectos del alcohol, acciones surgidas de la publicidad y popularidad. [1] Estas sustancias realmente no aportan energía, es decir, no brindan elementos que puedan usarse para ejecutar una actividad.

Se componen de distintas sustancias que actúan como estimulantes y como resultado provocan que el individuo se sienta con incremento de energía. Por medio de este estimulo incrementan de forma transitoria. Los niveles de concentración de catecolaminas (hormonas de alerta), generando de esta manera un mayor potencial para generar algún tipo de trabajo en un determinado tiempo. [2] Es por eso que buscamos informar y concientizar a la población sobre la importancia de la disminución del consumo de este, así como los efectos adversos que conlleva el abuso de esta sustancia en un lapso de tiempo. $[1,2]$

\section{Introducción a las bebidas energizantes}

Las bebidas energizantes son bebidas analcohólicas, generalmente gasificadas, compuestas básicamente por cafeína e hidratos de carbono (azúcares diversos de distinta velocidad de absorción), aminoácidos, vitaminas,

${ }^{1}$ Lic. Médico Cirujano, Universidad Autónoma de Estado de Hidalgo, Escuela superior de Tepeji del Rio, Av. Del Maestro No. 41 Colonia Noxtongo $2^{a}$ Sección, Tepeji del Rio, Hidalgo, México. Email: carmonajocelyn23@gmail.com, 2 almad.castillog.14k@gmail.com, 3 kinata97@gmail.com, 4 mont_ranita@hotmail.com. 
minerales, extractos vegetales, acompañados de aditivos acidulantes, conservantes, saborizantes y colorantes.

Según la definición de la tecnóloga de alimentos Melgarejo M. se las puede considerar: [3]

"Alimentos funcionales"

Ya que han sido diseñadas para proporcionar un beneficio específico: el de brindar al consumidor una bebida que le ofrezca aliviar la fatiga y mejorar condiciones cognitivas ante episodios de estrés.

Para algunos investigadores deberían llamarse "estimulantes" y no de energizantes, ya que una bebida energizante es aquella que se utiliza para aportar energía al cuerpo. En cambio, en estas bebidas utilizando el término de "energía" se refiere a cierto efecto estimulante de algunas sustancias en el organismo y no de un aporte calórico a partir de sus nutrientes.

El comienzo de la bebida energizante fue en año de 1906 con las llamadas gaseosas colas, su popularidad inicio con el ingrediente que lo componía que era la cafeína. Al paso de 20 años en el Reino Unido, William Owen crea una nueva bebida energizante para ayudar a los pacientes efectos, brindándoles energía, y es en año de 1938 cuando se empieza a vender en diferentes empresas. En Asia en el año de 1960 estas bebidas seguían cambiado con el objetivo de aliviar el cansancio. Posteriormente en el año de 1962 se empieza a comercializar una bebida energizante en, la cual contenía taurina gingen, niacina y vitaminas de complejo B. Durante ese mismo tiempo en Tailandia se fabricó otra bebida que aparte de contener esas sustancias se le agrego azúcar y cafeínas. [4]

Las industrias de bebidas colas comenzaron a crear nuevos productos los cuales contenían concentraciones elevadas de cafeína y azúcar, esto sucedió en los años de 1970 y 1980, y así mismo aparece en África la bebida energética más sobresaliente en ese momento, es ahí cuando se expande hasta Estados Unidos para que finalmente se comercialice globalmente. [5]

\section{3. "Red Bull"}

Bebida de origen austriaco creado por Dietrich Mateschits en 1980. Creo la fórmula de la bebida energizante "Red Bull energy Drinks" actualmente su venta abarca 171 países y se han consumido alrededor de 68000 millones de latas. [6]

\subsection{Componentes principales de las bebidas energéticas}

Cafeína: su origen puede ser original o sintético su efecto dura 4 o 5 horas.

Bloquea receptores de adenosina aumentando la cantidad de sinapsis y participa en la concentración celular de calcio, en resultado a esto es un fuerte estimulador del sistema nervioso central y del musculo esquelético, así como estimula la función cardiaca, la dilatación coronaria y relajación del musculo liso. [7]

Interviene en efectos del estado de ánimo, incrementa la energía física, provoca el estado de vigilia y mejora procesos cognitivos.

Tiende ser una sustancia adictiva y por ende su disminución de ingesta o suspensión conlleva a un síndrome de abstinencia. [8]

Glucosa: relacionado a la actividad neuronal ya que el cerebro para su metabolismo depende de los niveles de glucosa, esto en consecuencia señala que los cambios de concentraciones de glucosa en el organismo afectan la función neurológica.

La glucosa además puede disminuir la fatiga periférica en el sistema muscular y circuitos de repetición. [8]

Glucoronolactona: se forma en el hígado, no es una sustancia biodisponible, se adquiere de plantas. [10,11]

Taurinas (Acido 2-amino-etano-sulfonico): Aminoácido existente en el organismo y alimentos (leche materna, carnes y vegetales), este se difiere del resto de los aminoácidos porque no se incorpora a las proteínas, sino, es libre y abunda en los músculos, plaquetas y sistema nervioso. [9]

Se sabe que, durante el exceso de actividad física o estrés, los niveles de la taurina (reservas) disminuyen. Puede funcionar como neurotransmisor, regula la concentración de $\mathrm{Na}$ y $\mathrm{H} 2 \mathrm{O}$ en células, modula la excitabilidad neuronal y es un osmo regulador. También se adhiere a sales biliares y potencia la digestión de grasas, ya que es un componente de la bilis.

\section{Efectos adversos}

La vulnerabilidad a la intoxicación es mayor en personas que no consumen con frecuencia o no han consumido esta bebida. La ingesta de cafeína puede tener efectos adversos para la salud en cantidades altas. Los efectos adversos más frecuentes de la cafeína son palpitaciones, taquicardia, molestias gástricas, temblor, nerviosismo e insomnio. Dosis elevadas puede generar intensa ansiedad, miedo y crisis de angustia.

Es por eso que el abuso de bebidas energizantes puede generar alteraciones en la conducta que signifiquen un riesgo para la salud.

\section{Hipótesis}


Comprobar los efectos que generan las bebidas energizantes en cada individuo tomando en cuenta aspectos como dosis, estado de salud de quien lo ingiere, para poder determinar los cambios fisiológicos que se producen al consumir esta sustancia, para así poder informar y prevenir a la población sobre los riesgos que conlleva el uso y abuso de la bebida energizante "Red Bull".

\section{Conclusiones}

Con base a lo estudiado y experimentado sobre las bebidas energizantes en este caso con "Red Bull" podemos llegar a la conclusión de que al ingerir esta bebida tiene diferentes cantidades ocasionan alteraciones en el organismo del individuo, y en exceso puede tener graves consecuencias, incluso la muerte. Es por eso que debemos prevenir su consumo restringiendo su venta en personas menores, así como prevenir informando a la población sobre su ingesta y efectos en la salud.

\section{Agradecimientos}

Queremos agradecer al doctor Tangirala Venkata Krishna Karthik, por haber revisado de forma crítica nuestro artículo, así como hacernos observaciones para mejorar nuestra investigación. Al igual agradecer a todos nuestros compañeros por haber revisado y hecho críticas en cuanto a mejora de redacción en nuestro trabajo.

\section{Bibliografía}

[1] Itany M, Diab B, Riachidi S, Awada S, Al Hajje A, Bawab W, et al. Comsuption of energy drinks among lebanese youth: a pilot study on the prevalence and side effects. Int J High Risk Behav Addic. 2014; 3:e18857. 2. Doi: 10.58 12/ijhrba. 18857.eCollection 2014

[2] Friis K, Lyng JI, Lasgaard M, Larsen FB. Energy drinks consumption and the relation to socio-demographic factors and health behaviour among young adults in Denmark. A population-based study. Eur J Public Health. 2014; 24:840-4.

[3] Melgarejo Marta. El verdadero poder de las bebidas energéticas. Revista Énfasis Alimentación $\mathrm{N}^{\circ} 6$, diciembre 2004.

[4] Hamowy R. Government and public health in America. In: Limited EEP, ed. Government and public health in America. London: Edward Elgar Publishing; 2007. p. 140-2.

[5] Roberson J. Fight!! Ippatsu!! "Genki" energy drinks and the marketing of masculine indeology in Japan. Men Mascul. 2005;7:365-84

[6] Crecen las bebidas energizantes http://www.nutrar.com/detalle.asp?ID=4503.

[7] Haller CA, Jacob P 3rd, Benowitz NL. Pharmacology of ephedra alkaloids and caffeine after single-dose dietary supplement use. Clin Pharmacol Ther. 2002 jun

[8] Specterman M, Bhuiya A, Kuppuswamy A, Strutton PH, Catley $M$, Davey NJ. The effect of an energy drink containing glucose and caffeine on human corticospinal excitability. Physiol Behav. 2005 Jan 17; 83(5):723-8.

[9] Finnegan D. The health effects of stimulant drinks. Nutrition Bulletin 2003 Jun; 28 (2): 147-155.

[10] Horne JA, Reyner LA. (2000). "Beneficial effects of an "energy drink" given to sleepy drivers". Amino Acids 2001; 20(1):83-9.
[11] Pasiakos SM, Petrancosta R, Wygand J, Otto RM. The Effects of a Commercial Energy Drink on Repeated High Intensity Anaerobic Cycling Performance. Med Sci Sports Exerc 2005 May. 37(5) Suppl S42.

Haga clic o pulse aquí para escribir texto. 